\title{
Application of two classifiers fusion based on support vector machines method and time series comparison DTW to recognize maritime objects upon FLIR images
}

Tadeusz Pietkiewicz

Tadeusz Pietkiewicz, "Application of two classifiers fusion based on support vector machines method and time series comparison DTW to recognize maritime objects upon FLIR images," Proc. SPIE 11442, Radioelectronic Systems Conference 2019, 114420 U (11 February 2020); doi: $10.1117 / 12.2565063$ 


\title{
Application of two classifiers fusion based on support vector machines method and time series comparison DTW to recognize maritime objects upon FLIR images
}

\author{
Tadeusz Pietkiewicz ${ }^{*}$ \\ Institute of Radioelectronics, Military University of Technology \\ Gen. S. Kaliskiego str. 2, 00-908 Warszawa, POLAND
}

\begin{abstract}
Infrared image recognition by means of FLIR cameras (forward-looking infrared) is one of the elements of the recognition of the maritime situation and it supports in many situations the creation of so-called maritime picture. This paper presents results of two FLIR image classifiers research. The first part presents the use of SVM (Support Vector Machine) to classify images of maritime objects, while the second part presents the classifier using the time series comparison method DTW (data time warping). The SVM network uses to perform the multi-class classification the oneagainst-all method. Both classifiers use the histograms of vertical projection of pre-processed FLIR images as input data (for training and testing). These histograms are created as a result of FLIR color images processing, including, among others, transformation of color images into grayscale images, grayscale images segmentation using the Otsu algorithm with a possible manual correction, rescaling, centering and leveling. In the further part of the work a method of determining the basic belief assignment is proposed for both SVM and DTW classifiers. In the final part of the paper test results of the both classification methods and their fusion by the Dempster's method for a set of maritime objects FLIR images registered in the Baltic Sea are presented.
\end{abstract}

Keywords: FLIR images recognition, image classifiers, SVM networks, time series comparison, data time warping, basic belief assignment, the Dempster's method of information fusion

\section{INTRODUCTION}

FLIR (forwared looking infra-red) passive infrared sensors are used for short- to medium-ranged recognition from 2 to 20 nautical miles depending on the size of the object being recognized and the conditions of observation. They are mounted on maritime and air platforms in the armed forces and border guards of many countries. FLIR cameras create a monochrome image in which the luminance of each pixel is proportional to the temperature of the observed point. These cameras often artificially color the image to present it to the operator. The method of assigning the color to the temperature is usually shown on the image. The natural way is to assign higher temperatures to yellow colors, and the lowest temperatures to blue and purple colors. From the point of view of image recognition, these colors are artificial and should be removed from the image and converted into shades of gray.

Recognition of maritime objects based on FLIR images should first answer the question whether the registered object is a maritime object. If one gets a positive answer, one expects an answer to the next question, whether the object being recognized belongs to one of the classes from the training set (previously recorded and classified images), or possibly state the inability to recognize the type.

FLIR images can be distorted due to specific atmospheric conditions (fog or rain) and solar lighting containing infrared radiation as well as due to physical processes taking place in the camera.

The geometry of the silhouettes of maritime objects can be changed as a result of changing camera settings, different distance of the object from the camera and different object observation angles (so-called aspect angles).

The above-mentioned factors make the process of recognizing maritime objects based on FLIR images a multi-stage process.

* tadeusz.pietkiewicz@wat.edu.pl;phone 48261 837513; fax 48261 837461; wel.wat.edu.pl

Radioelectronic Systems Conference 2019, edited by Piotr Kaniewski, Jan Matuszewski, Proc. of SPIE Vol. 11442, $114420 \mathrm{U} \cdot$ (C) 2020 SPIE · CCC code: 0277-786X/20/\$21 · doi: 10.1117/12.2565063 
The process of comparing and recognizing objects takes into account their specific features called distinctive features. The choice of features is related to the specifics of the recognized objects, the method of recording images, and the methods and recognition algorithms used. In the case of recognition of maritime object images, the basic element of the image being analyzed is the silhouette of this object. It can be characterized by various sets of distinctive features. The principal component analysis (PCA) method or methods using deep neural networks take into account the luminance of all silhouette pixels. The method of compressing information about the silhouette called the normalized histogram of the vertical brightness projection was used in the paper. The histogram of the vertical brightness projection of the maritime object silhouette allows to use the DTW classifier in a natural way, while in the case of the SVM classifier it allows reducing the dimensionality of the classification task.

Similar topics were discussed in many papers, with different classification methods $[7,9,12,13,15,16,27,29]$.

Two methods of classifying of maritime object silhouettes are used in the paper: a method based on multiclass classification using a support vector network SVM (SVM - Support Vector Machine) $[1,2,3,4,5,6]$ called the SVM classifier in the paper and a DTW method of comparing time series (Date Time Warping), called the DTW classifier $[17,18,21,29]$.

The first classification method is based on SVM classifiers, which in the literature are also called SVM networks because of their similarity to neural networks. These classifiers are, by their very nature, two-class classifiers, and thus they allow finding the answer to the question whether the recognized object belongs to one of two classes. In real problems, the set of patterns is usually multi-class, hence methods for using two-class classifiers for multi-class classification have been developed. One of such methods called "one against all" is used in the paper. It has been modified for the fusion of SVM and DTW classifiers in such a way that it is possible to determine the basic belief assignment over the set of pattern types. The classifier can operate directly on the space of maritime object silhouette images, however in the paper, due to the consistency of considerations, one assumed that the SVM classifier, like the DTW classifier, operates in the space of the vertical brightness projection histograms.

Another problem to be addressed was the assessment of the linear separability of the training set histograms. It is related to the possible transformation of the original space of distinctive features into a space with a much larger number of dimensions and the use of the appropriate kernel function of the transformation. In the paper, a hint contained in [4] is used. It allows the use of a linear kernel when the number of patterns in the training set is much smaller than the number of distinctive features of the patterns.

The second recognition method used in the paper is the classification by the DTW time series comparison method, described in detail in $[17,18,21,29]$.

The DTW method determines the distance of the histogram of the vertical brightness projection of the recognized image from the average histogram of the vertical brightness projection of each type of maritime object included in the training set (the pattern database). For this it uses the dynamic programming method of R. Bellman. The DTW method determines the distance of two time series in a situation where the time course of one of the series has been deformed (stretched or compressed).

Both classifiers require FLIR images to be previously processed, preparing a histogram of the vertical brightness projection. The purpose of this process is, among other things, to eliminate unnecessary information about the background of the object and interference, as well as to normalize the silhouette of the object. The image pre-processing process may include segmentation, brightness normalization, silhouette scaling, silhouette centering, silhouette leveling and extraction of distinctive features. Some problems of information pre-processing have been presented in [11,20,29].

One of the important objectives of the work was to evaluate the effectiveness of information fusion methods [22] applied to the results of SVM and DTW classifiers. In the paper, methods for determining the basic belief assignment [22] on a set of possible decisions of both classifiers were proposed.

In the final part of the paper, the results of tests of two classifiers SVM and DTW and the results of the fusion of these classifiers using the Dempster's rule [22] are presented. 


\section{VERTICAL BRIGHTNESS PROJECTION HISTOGRAM AS A VECTOR OF DISTINCTIVE FEATURES OF A MARITIME OBJECT}

As mentioned in chapter 1 in this paper, it was assumed that the set of distinctive features of the object is a histogram of the vertical brightness projection (vector), which is formed in the process of initial processing, segmentation and secondary processing. One should note that it significantly reduces the dimensionality of the classification problem. In the paper, this vector has a size of 640. If all the pixels of the original image were used, the dimensionality of the problem would be $640 \times 480=307,200$.

The histogram $\boldsymbol{L} \boldsymbol{V}$ of the vertical brightness projection is determined as a result of summing the brightness of the pixels in each column and is expressed by the following formula:

$$
\boldsymbol{L} \boldsymbol{V}(j)=\sum_{i=1}^{h} \boldsymbol{B}(i, j) \text { for } j \in\{1, \ldots, w\},
$$

where:

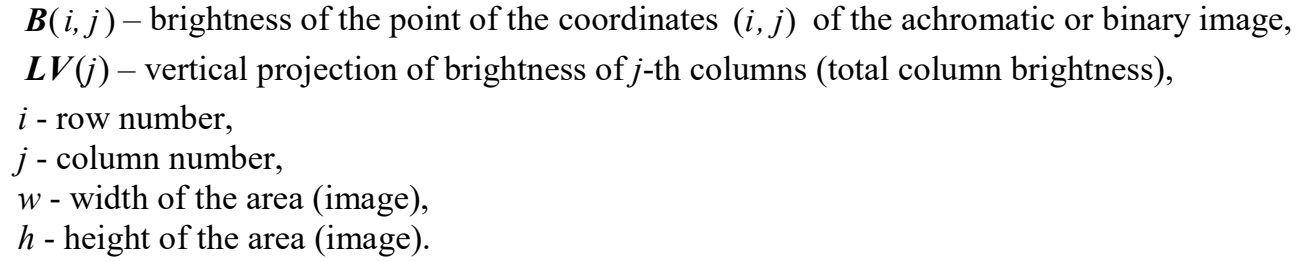

\section{CHARACTERISTICS OF SVM CLASSIFIER}

\subsection{Introduction}

Support Vector Machine SVM is a useful technique for data classification [1,2,3,4,5,6,23,25,26]. The SVM machine in the Polish literature on the subject is most often called the SVM network due to its simple interpretation using neural networks. First, basic information explaining the principles of data classification using SVM in the case of binary (twoclass) classification will be presented. This technique will then be extended to the problem of multi-class classification.

\subsection{Binary classification}

The SVM classifier belongs to the set of classifiers that maximize the separation margin $[1,2,3]$ The SVM classifier belongs to the set of classifiers that maximize the separation margin. These classifiers recognize patterns belonging to two classes by specifying a decision surface that provides maximum distance to the nearest points in the training set called support vectors.

Let us assume that a set of training pairs is given $\left(\boldsymbol{x}_{i}, y_{i}\right)$ for $i=1, \ldots, p$, wherein each point $\boldsymbol{x}_{i} \in \boldsymbol{R}^{n}$ belongs to one of two classes of patterns identified by labels $y_{i}=+1$ (class 1) or $y_{i}=-1$ (class 2). Assuming a linear separability of classes, the equation of the separating hyperplane can be written using the formula

$$
f(\boldsymbol{x})=\boldsymbol{w}^{T} \boldsymbol{x}+b=0,
$$

where $\boldsymbol{w}=\left[w_{1}, w_{2}, \ldots, w_{N}\right]^{T}$ is an $N$-dimensional weight vector and $\boldsymbol{x}=\left[x_{1}, x_{2}, \ldots, x_{N}\right]^{T}$ is a vector of the distinctive features values of the object. The $b$ value specifies the hyperplane offset relative to the origin of the coordinate system.

Decision equations of classification take the following form:

$$
\begin{array}{lll}
\boldsymbol{w}^{T} \boldsymbol{x}_{i}+b>0 & \text { for } & y_{i}=1 \\
\boldsymbol{w}^{T} \boldsymbol{x}_{i}+b<0 & \text { for } & y_{i}=-1
\end{array} .
$$




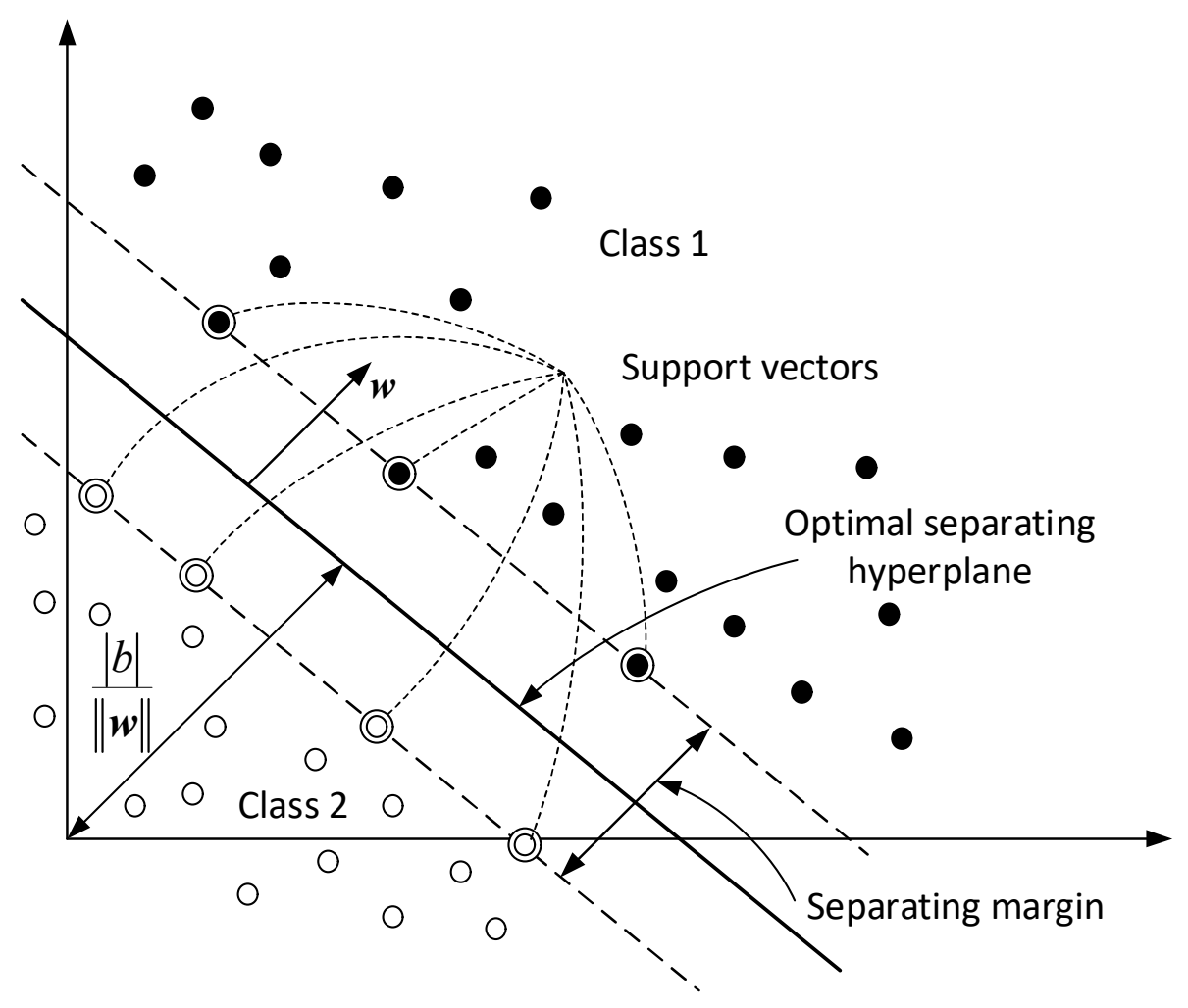

Figure 1. Optimal separating hyperplane and separating margin in SVM in a two-dimensional space.

The distance of any point $\boldsymbol{x}$ from the hyperplane (2) is

$$
d(\boldsymbol{x})=\frac{\left|\boldsymbol{w}^{T} \boldsymbol{x}+b\right|}{\|\boldsymbol{w}\|}=\frac{|f(\boldsymbol{x})|}{\|\boldsymbol{w}\|} .
$$

Intuitively, one can say that the greater the distance (4) of point $x$ from the hyperplane (2), the greater the reliability of the classification.

Because the assumption of the linear separability of the training data has been made, so no training data satisfies the equation $\boldsymbol{w}^{T} \boldsymbol{x}_{i}+b=0$. It follows that the width of the separation margin is greater than zero, what means that inequalities (3) after their normalization can be written in the following form

$$
\begin{array}{lll}
\boldsymbol{w}^{T} \boldsymbol{x}_{i}+b \geq 1 & \text { for } & y_{i}=1 \\
\boldsymbol{w}^{T} \boldsymbol{x}_{i}+b \leq-1 & \text { for } & y_{i}=-1
\end{array} .
$$

One can write these two inequalities in one formula

$$
y_{i}\left(\boldsymbol{w}^{T} \boldsymbol{x}_{i}+b\right) \geq 1 .
$$

If a pair $\left(\boldsymbol{x}_{i}, y_{i}\right)$ satisfies in (6) equality, then the vector $\boldsymbol{x}_{i}$ is called a support vector SV.

Assuming a linear separability of training data, these vectors only decide on the location of the optimal separation hyperplane and the width of the separation margin, which has a value

$$
d(\boldsymbol{x})=\frac{2}{\|\boldsymbol{w}\|} .
$$

Optimal separating hyperplane (2) and separating margin in SVM in a two-dimensional space are presented in Figure 1. 
The task of optimal separation margin design is to find such a margin, which has a maximum width. The problem of optimal selection of the separation hyperplane and the separation margin width comes down to solving the quadratic programming task in the following form

$$
\min _{\boldsymbol{w}, b} \frac{1}{2} \boldsymbol{w}^{T} \boldsymbol{w}
$$

with constraints

$$
y_{i}\left(\boldsymbol{w}^{T} \boldsymbol{x}_{i}+b\right) \geq 1 .
$$

This is a quadratic programming task with constraints that can be solved by the Lagrange multipliers method with $\alpha=\left[a_{1}, \alpha_{2}, \ldots, \alpha_{N}\right]^{T} \geq 0$ multipliers using Karush-Kuhn-Tucker conditions [28]. Lagrange function is as follows

$$
L(\boldsymbol{w}, b, \boldsymbol{\alpha})=\frac{1}{2} \boldsymbol{w}^{T} \boldsymbol{w}-\sum_{i=1}^{N} \alpha_{i} y_{i}\left(\boldsymbol{w}^{T} \boldsymbol{x}_{i}+b\right)+\sum_{i=1}^{N} \alpha_{i} .
$$

The solution to this optimization task is as follows $[1,2,3]$

$$
\boldsymbol{w}=\sum_{i=1}^{N} \alpha_{i} y_{i} \boldsymbol{x}_{i}
$$

wherein non-zero Lagrange multipliers correspond only to support vectors.

To determine the constant value $b$, one can use the fact that, according to Karush-Kuhn-Tucker conditions at the saddle point of the Lagrange function, the product of the multiplier by the constraint associated with the support vector $\boldsymbol{x}_{s v}$ is zero [28] $\alpha_{s v}\left(\boldsymbol{w}^{T} \boldsymbol{x}_{s v}+b \pm 1\right)=0, \alpha_{s v}>0$. From here one can receive

$$
b=-\boldsymbol{w}^{T} \boldsymbol{x}_{s v} \pm 1
$$

The equation of the optimal separation hyperplane is as follows

$$
\sum_{i=1}^{N} \alpha_{i} y_{i} \boldsymbol{x}_{i}^{T} \boldsymbol{x}+b=0,
$$

while the decision function is as follows

$$
\left\{\begin{array}{l}
f(\boldsymbol{x})=\sum_{i=1}^{N} \alpha_{i} y_{i} \boldsymbol{x}_{i}^{T} \boldsymbol{x}+b \geq 1 \quad \rightarrow \quad y=1 \\
f(\boldsymbol{x})=\sum_{i=1}^{N} \alpha_{i} y_{i} \boldsymbol{x}_{i}^{T} \boldsymbol{x}+b \leq-1 \quad \rightarrow \quad y=-1
\end{array} .\right.
$$

More complex models of SVM linear networks include the possibility of incompletely linearly separable training data. Suitable formulas can be found in $[1,2,3,6]$.

In the above considerations, a linear separability of training data was assumed. The linear inseparability of training data does not mean a lack of their separability at all. A common solution is the non-linear projection of original data into another functional space in which transformed patterns are linearly separable or the probability of their separability is very high. The condition is the use of non-linear transformation with a sufficiently high dimension $K \gg N$ of the feature space. The above-mentioned construction of the separation hyperplane and decision rule can be applied in a new space that is specified by the projection function $\Phi$. The key property of the projection function $\Phi$ is that the scalar product of vectors $\Phi\left(\boldsymbol{x}_{i}\right)^{T} \cdot \Phi(\boldsymbol{x})$ in the result space can be represented as a certain kernel function $K\left(\boldsymbol{x}_{i}, \boldsymbol{x}\right)$. The basic kernel functions are following:

- linear kernel: $K\left(\boldsymbol{x}_{i}, \boldsymbol{x}\right)=\boldsymbol{x}_{i}^{T} \boldsymbol{x}$,

- polynomial kernel: $K\left(\boldsymbol{x}_{i}, \boldsymbol{x}\right)=\left(\alpha \boldsymbol{x}_{i}^{T} \boldsymbol{x}+r\right)^{d}, \alpha>0$, 
- Gaussian kernel (RBF - radial basis function): $K\left(\boldsymbol{x}_{i}, \boldsymbol{x}\right)=\exp \left(-\beta\left\|\boldsymbol{x}_{i}-\boldsymbol{x}\right\|^{2}\right), \beta>0$,

- sigmoid kernel: $K\left(\boldsymbol{x}_{i}, \boldsymbol{x}\right)=\tanh \left(\gamma \boldsymbol{x}_{i}^{T} \boldsymbol{x}+r\right)$.

The values of $\alpha, \beta, \gamma, r$ and $d$ are the parameters of the kernels.

In [4] there are some guidelines regarding situations in which a linear kernel can be used and in which an RBF radial kernel can be used. If the number of patterns in the training set is much smaller than the number of distinctive features of the patterns, a linear kernel can be used. In this paper, a linear kernel was used, because the number of training patterns was 35 ( 5 images for 7 types of patterns), and the length of the distinctive features vector was 640 .

The equation of the separating hyperplane after applying the transformation of the primary space of distinctive features by means of the kernel function is as follows

$$
\sum_{i=1}^{N} \alpha_{i} y_{i} K\left(\boldsymbol{x}_{i}^{T}, \boldsymbol{x}\right)+b=0
$$

while the decision function is as follows

$$
\left\{\begin{array}{lll}
f(\boldsymbol{x})=\sum_{i=1}^{N} \alpha_{i} y_{i} K\left(\boldsymbol{x}_{i}^{T}, \boldsymbol{x}\right)+b \geq 1 & \rightarrow & y=1 \\
f(\boldsymbol{x})=\sum_{i=1}^{N} \alpha_{i} y_{i} K\left(\boldsymbol{x}_{i}^{T}, \boldsymbol{x}\right)+b \leq-1 & \rightarrow & y=-1
\end{array} .\right.
$$

\subsection{Multiclass classification}

SVM networks divide data into two classes. Unlike classic neural networks, where we can have multiple outputs (each output is associated with one class), recognition of multiple classes requires the implementation of multiple classification tasks using multiple SVM networks. The best-known strategies for solving the problem of multi-class classification are "one - against - one" and "one - against - all" methods $[1,24,26]$. Suppose the training base has M types of patterns.

In the case of the "one-against-one" method, $M(\mathrm{M}-1) / 2$ SVM classifiers are constructed. They distinguish sequentially two classes from the training set. One can receive a decision function for each pair of classes $i$ and $j$

$$
f_{i j}(\boldsymbol{x})=\boldsymbol{w}_{i j}^{T} \Phi(\boldsymbol{x})+b_{i j} \quad i, j \in \boldsymbol{M}=\{1, \ldots, M\}, i \neq j .
$$

After training all SVM networks, you can proceed to classify objects from the test set. If $\operatorname{sgn}\left(f_{i j}(\boldsymbol{x})\right)$ indicates the $i$-th class, one should increase by 1 the counter of this class indications, in the opposite case it should be increased by 1 the counter of the $j$-th class. Finally, we choose the class whose counter has reached the highest value.

In the case of the "one - against - all" method, $M$ SVM classifiers are constructed, each network being trained on a different training set. Suppose we train the $m$-th SVM two-class network. Class 1 includes $m$-th type patterns, while class 2 includes other types. Finally, we receive a decision function for each network

$$
f_{m}(\boldsymbol{x})=\boldsymbol{w}_{m}^{T} \Phi(\boldsymbol{x})+b_{m} \quad m \in \boldsymbol{M}=\{1, \ldots, M\} .
$$

After training all SVM networks, one can proceed to classify objects from the test set. If $\operatorname{sgn}\left(f_{i j}(\boldsymbol{x})\right)$ indicates the $m$-th class, one should increase the number of indications in this class by 1, and in the opposite case one should increase the number of indications by 1 for all classes in the combined class. Finally, we choose the class whose counter achieved the highest value of wins. The authors [26] prefer the "one - against - all" method because of the linear dependence of the number of SVM networks on the number of pattern types in the training set.

The possibility of another extended interpretation of the results obtained by the "one - against - all" method is presented in [24]. According to [26], the higher the value of the function $f_{m}(\boldsymbol{x})(18)$, the more reliable the classification result is. In the case of a linear kernel, such a criterion of reliability may be the distance of the recognized object from the separation plane, which is equal 


$$
f_{m}(\boldsymbol{x})=\boldsymbol{w}_{m}^{T} \Phi(\boldsymbol{x})+b_{m} \quad m \in \boldsymbol{M}=\{1, \ldots, M\} .
$$

In point 5.3 of the paper, the value of the $f_{m}(\boldsymbol{x})$ decision function was used to construct the basic belief assignment on the results of the SVM multi-class classification.

In SVM multi-class classification, each $m$-th classifier determines the value of its decision function $f_{m}(\boldsymbol{x})$.

Considering the classification results as a whole, one of three situations may occur:

1. Only one $f_{m}(\boldsymbol{x})$ has a positive value, and all the others are negative. In this case, the number of positive classifier specifies the pattern type number.

2. More than one of the $f_{m}(\boldsymbol{x})$ are positive. If we assume that the higher the value of the decision function, the more reliable the classification result is, then the number of the classifier corresponding to the highest value of the function $f_{m}(\boldsymbol{x})$ determines the number of the pattern type, what can be written as follows

$$
m^{*}=\arg \max _{m \in\{1, \ldots, M\}, f_{m}(x)>0} f_{m}(\boldsymbol{x}),
$$

where $i d$ is the number of the recognized type.

3. None of the $f_{m}(\boldsymbol{x})$ values is positive. That should be regarded as the new image belongs to a maritime object which type is not included in the training set (unknown object).

\section{CHARACTERISTICS OF DTW CLASSIFIER}

The DTW (Dynamic Time Warping) time series comparison method defines a class of algorithms for comparing series (sequences) of values. The DTW method allows determining the similarity of two time series assuming that both can describe different time intervals and the time variable can be locally compressed or stretched.

The purpose of the DTW method is to compare two sequences (most often dependent on time and thus called time series)

$$
\boldsymbol{L} \boldsymbol{V}_{1}=\left\{x_{1}, x_{2}, \ldots, x_{N}\right\}
$$

of length $N \in \mathcal{N}$ and

$$
\boldsymbol{L} \boldsymbol{V}_{2}=\left\{y_{1}, y_{2}, \ldots, y_{M}\right\}
$$

of length $M \in \mathcal{N}$.

These series may be discrete signals or generally the sequences of values of certain features measured at equal intervals.

The DTW method allows to determine the distance $D T W\left(\boldsymbol{L} \boldsymbol{V}_{1}, \boldsymbol{L} \boldsymbol{V}_{2}\right)$ of two time series based on a matrix $\boldsymbol{D}$ of crossdistances between all combinations of pairs of sequence elements $\boldsymbol{L} \boldsymbol{V}_{1}$ and $\boldsymbol{L} \boldsymbol{V}_{2}$. The works [17,18,19] contain a detailed description of the DTW method.

The DTW classifier determines the distance between the histogram of the vertical brightness projection of the recognized object and the average histogram of the vertical brightness projection of the objects belonging to the cluster of a given type in the training set.

Let us assume the following notation:

$\overline{\boldsymbol{L V}}_{k}$ - the average brightness projection histogram of $k$-th type of image in the training set,

$\boldsymbol{L} \boldsymbol{V}$ - the brightness projection histogram of the recognized image,

$D T W\left(\boldsymbol{L} \boldsymbol{V}, \overline{\boldsymbol{L V}}_{k}\right)$ - the distance between the brightness projection histogram of a recognized silhouette and the average brightness projection histogram of $k$-th type of silhouette in the training set. 
The object recognition task consists in specifying the image type number $k^{*}$ in the pattern database that:

$$
k^{*}=\arg \min _{k \in P T N S} D T W\left(\boldsymbol{L} \boldsymbol{V}, \overline{\boldsymbol{L}}_{k}\right),
$$

where $\boldsymbol{P T N S}$ is a set of image pattern type numbers.

\section{FUSION OF INFORMATION FROM SVM AND DTW CLASSIFIERS}

\subsection{The process of fusion of information from two classifiers}

Each classifier used in the work transfers to the fusion module a vector of measures of reliability distribution on a set of types of objects in the training set. In this paper, it was assumed that both classifiers have the same training set. The set of possible hypotheses related to individual types of objects is as follows

$$
\Theta=\left\{\theta_{i}, i=1, \ldots, P T N\right\},
$$

wherein the index $i$ numbers the type of the maritime object whose images are stored in a training set. PTN is the number of pattern types.

The hypotheses are mutually exclusive, i.e.

$$
\theta_{i} \cap \theta_{j}=\left\{\begin{array}{l}
\theta_{i}, \text { if } i=j, \\
\varnothing, \text { if } i \neq j .
\end{array}\right.
$$

Each classifier sends its decisions in the form of a bba measure vector (bba - basic belief assignment).

$$
\boldsymbol{m}_{i}=\left[m_{i}\left(\theta_{1}\right), \ldots, m_{i}\left(\theta_{P T N}\right)\right],
$$

wherein the index $i=1$ determines the bba measure vector calculated by the SVM classifier and the index $i=2$ determines the bba measure vector calculated by the DTW classifier.

The information fusion procedure is described by the following formula:

$$
\boldsymbol{m}_{F}=R_{F}\left(\boldsymbol{m}_{1}, \boldsymbol{m}_{2}\right) \text {, }
$$

wherein $\boldsymbol{m}_{F}$ is a vector of the vector of bba masses determined by the $R_{F}$ information fusion rule based on the vectors $\boldsymbol{m}_{1}$ and $\boldsymbol{m}_{2}$ of bba masses.

In the further part of the paper the Dempster's rule [22] is described as a combination of the bba masses vectors. It has been used to implement the information fusion of two classifiers SVM and DTW.

\subsection{The Dempster's rule of combination of bba vectors}

The Dempster's rule of the bba measure vectors $\boldsymbol{m}_{i}$ sent by the classifiers is described for each $\theta_{j} \in \boldsymbol{\Theta}$ by the following formula [22]:

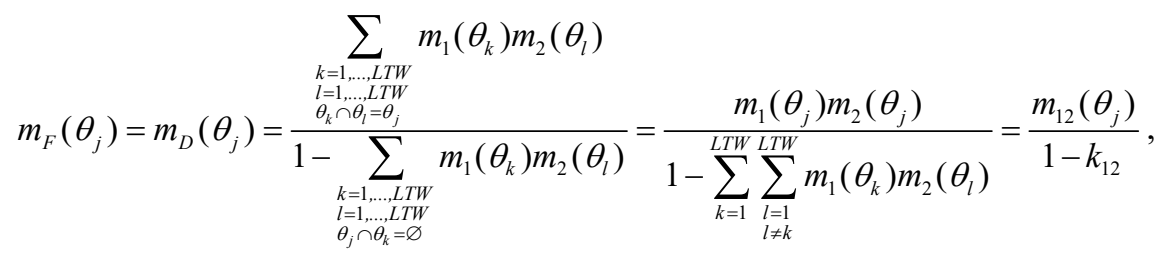

wherein the $k_{12}$ degree of conflict is defined by the formula:

$$
k_{12}=\sum_{\substack{k=1, \ldots L L W \\ l=1, \ldots L, L W \\ \theta_{k} \cap \theta_{l}=\varnothing}} m_{1}\left(\theta_{k}\right) m_{2}\left(\theta_{l}\right)=\sum_{k=1}^{L T W} \sum_{\substack{l=1 \\ l \neq k}}^{L T W} m_{1}\left(\theta_{k}\right) m_{2}\left(\theta_{l}\right),
$$


while

$$
m_{12}\left(\theta_{j}\right)=m_{1}\left(\theta_{j}\right) m_{2}\left(\theta_{j}\right) .
$$

Formula (28) makes sense if the denominator of expression (28) is nonzero, i.e. the degree of conflict $k_{12}$ is less than 1.

\subsection{The method of determining the bba for the SVM classifiers}

The procedure of image recognition by means of the SVM method "one - against - all" in accordance with the content of point 3.3 and [24.26] allows to determine the basic belief assignment bba on a set of pattern types. Each $k$-th pattern type is associated with one SVM and the identification process determines the value of the decision function

$$
f_{k}(\boldsymbol{x})=\sum_{i=1}^{N_{k}} \alpha_{i}^{k} y_{i}^{k} K\left(\boldsymbol{x}_{i}^{k T}, \boldsymbol{x}\right)+b_{k} .
$$

The value of this function can be used to determine the value of the degree of belief that the recognized object belongs to the class with the number $k(k=1, \ldots, P T N)$. In [3] it was proposed to use the logistic regression function in accordance with the following formula

$$
m(\boldsymbol{x}, k)=\frac{e^{f_{k}(\boldsymbol{x})}}{1+e^{f_{k}(\boldsymbol{x})}} .
$$

In the formula (32) $k$ numbers SVMs. As one can see $0<m(x, k)<1$.

The above measure is not standardized, therefore it should be transformed in the following way

$$
\tilde{m}(\boldsymbol{x}, k)=\frac{m(\boldsymbol{x}, k)}{\sum_{i=1}^{P T N} m(\boldsymbol{x}, i)} .
$$

One should note that the above method of mass determination is simplified, because it does not take into account the lack of the type of image pattern corresponding to the recognized image.

\subsection{The method of determining the bba for the DTW classifiers}

The DTW image recognition procedures determines the distance between the values of the distinctive features of the recognized image and the values of the distinctive features of the image pattern types stored in the training set.

By accepting the designations in Chapter 4, one can determine the value of the degree of belief that the recognized object belongs to the class with the number $k(k=1, \ldots, P T N)$ as follows

$$
m(\boldsymbol{L} \boldsymbol{V}, k)=e^{-D T W\left(\boldsymbol{L} \boldsymbol{V}, \overline{\boldsymbol{L V}}_{k}\right)} .
$$

From formula (34), it follows that if $D T W\left(\boldsymbol{L} \boldsymbol{V}, \overline{\boldsymbol{L V}}_{k}\right)=0$, then $m(\boldsymbol{L} \boldsymbol{V}, k)=1$, while if $D T W\left(\boldsymbol{L} \boldsymbol{V}, \overline{\boldsymbol{L V}}_{k}\right)>0$, then $0<m(\boldsymbol{L} \boldsymbol{V}, k)<1$.

The above measure is not standardized, therefore it should be transformed in the following way

$$
\tilde{m}(\boldsymbol{L} \boldsymbol{V}, k)=\frac{m(\boldsymbol{L} \boldsymbol{V}, k)}{\sum_{i=1}^{P T N} m(\boldsymbol{L} \boldsymbol{V}, i)} .
$$

One should note that the above method of mass determination is simplified, because it does not take into account the lack of the type of image pattern corresponding to the recognized image.

An extended version of determining the basic belief assignment can also be considered. The image recognition classifier compares the distance $D T W\left(\boldsymbol{L} V, \overline{\boldsymbol{L V}}_{k}\right)$ with the permissible distance $\delta$ from the center of the cluster of the $k$-th type of image pattern. The distance $\delta$ defines the recognition area of the $k$-th image pattern. The set of image patterns will be 
divided into two subsets: patterns that meet the classification condition in relation to the recognized image $-\boldsymbol{D}_{x}^{+}$and patterns that do not meet the condition of classification in relation to the recognized image $-\boldsymbol{D}_{x}^{-}$.

The value of the basic belief assignment is then determined by the formula

$$
m(\boldsymbol{L} \boldsymbol{V}, k)=\left\{\begin{array}{lll}
e^{-D T W\left(\boldsymbol{L} V, \overline{\boldsymbol{L V}}_{k}\right)} & \text { for } & D T W\left(\boldsymbol{L} \boldsymbol{V}, \overline{\boldsymbol{L}}_{k}\right) \leq \delta \\
0 & \text { for } & D T W\left(\boldsymbol{L} \boldsymbol{V}, \overline{\boldsymbol{L V}}_{k}\right)>\delta
\end{array} .\right.
$$

The sum of the measures assigned to the images whose distinctive features lie outside the boundary will be treated as the measure assigned to the new hypothesis "unrecognized object".

\section{RESULTS OF MARITIME OBJECTS RECOGNITION USING SVM AND DTW CLASSIFIERS AND THE FUSION OF THESE CLASSIFIERS}

In the pattern database there were 35 FLIR images of 7 types of maritime objects. The image pre-processing, segmentation and secondary processing operations have been performed for all the images and the vertical brightness projection histogram has been determined.

This paper uses a different training set (a pattern database) than in [29], hence the results of the DTW classifier tests differ from the results contained therein.

\subsection{Results of maritime objects recognition for SVM classifier}

Research on the efficiency of maritime object recognition based on FLIR images using SVM classifiers was preceded by the process of teaching two-class SVM networks in accordance with the "one - against - all" strategy. The study consisted in selecting different images from the test database, and then performing as many classification tasks for each recognized object as the number of pattern types contained in the training set. The choice of the recognized type was carried out in accordance with the principles described in section 3.3 and the formula (20). It was also possible to obtain a result indicating that the object type was not recognized (point 3 therein), which was treated as an error, because all object types from the test set belonged to the set of object types from the training set. The results are shown in the Table 1.

Table 1. The results of recognition of maritime object images using the SVM classifier.

\begin{tabular}{|c||c|c|c|c||}
\hline $\begin{array}{c}\text { Maritime } \\
\text { object type } \\
\text { number }\end{array}$ & $\begin{array}{c}\text { Number of } \\
\text { tested images }\end{array}$ & $\begin{array}{c}\text { Number of correctly } \\
\text { recognized maritime } \\
\text { objects }\end{array}$ & $\begin{array}{c}\text { Number of incorrectly } \\
\text { recognized maritime } \\
\text { objects }\end{array}$ & $\begin{array}{c}\text { Mean value of correctly } \\
\text { recognized maritime } \\
\text { objects }\end{array}$ \\
\hline \hline 1 & 14 & 7 & 7 & $50,0 \%$ \\
\hline 2 & 28 & 25 & 3 & $89,3 \%$ \\
\hline 3 & 16 & 9 & 7 & $56,3 \%$ \\
\hline 4 & 18 & 10 & 8 & $55,6 \%$ \\
\hline 5 & 10 & 6 & 4 & $60 \%$ \\
\hline 6 & 15 & 15 & 0 & $100 \%$ \\
\hline 7 & 9 & 5 & 4 & $55,6 \%$ \\
\hline \hline Together & 110 & 77 & 33 & $70,0 \%$ \\
\hline
\end{tabular}

\subsection{Results of maritime objects recognition for DTW classifier}

The recognition procedure examined subsequently various images from the test data set. A cluster of such type of pattern images was chosen, which had the smallest DTW distance from the recognized object. If the type of image selected was 
consistent with the type of image recognized, one can assume that the image was recognized correctly, otherwise the image was recognized incorrectly. The results are presented in the Table 2

Table 2. The results of recognition of maritime object images using the DTW classifier.

\begin{tabular}{|c||c|c|c|c||}
\hline $\begin{array}{c}\text { Maritime } \\
\text { object type } \\
\text { number }\end{array}$ & $\begin{array}{c}\text { Number of } \\
\text { tested images }\end{array}$ & $\begin{array}{c}\text { Number of correctly } \\
\text { recognized maritime } \\
\text { objects }\end{array}$ & $\begin{array}{c}\text { Number of incorrectly } \\
\text { recognized maritime } \\
\text { objects }\end{array}$ & $\begin{array}{c}\text { Mean value of correctly } \\
\text { recognized maritime } \\
\text { objects }\end{array}$ \\
\hline \hline 1 & 14 & 14 & 0 & $100 \%$ \\
\hline 2 & 28 & 22 & 6 & $78,6 \%$ \\
\hline 3 & 16 & 16 & 0 & $100 \%$ \\
\hline 4 & 18 & 12 & 6 & $66,7 \%$ \\
\hline 5 & 10 & 10 & 0 & $100 \%$ \\
\hline 6 & 15 & 7 & 8 & $46,7 \%$ \\
\hline 7 & 9 & 4 & 5 & $44,4 \%$ \\
\hline \hline Together & 110 & 85 & 25 & $77,3 \%$ \\
\hline
\end{tabular}

\subsection{Results of maritime objects recognition by means of Dempster's fusion of SVM and DTW classifiers}

The results of using the Dempster's rule to fuse information of two SVM and DTW classifiers are presented in the Table 3 .

Table 3. The results of recognition of maritime object images by means of Dempster's fusion of SVM and DTW classifiers.

\begin{tabular}{|c|c|c|c|c||}
\hline $\begin{array}{c}\text { Maritime object } \\
\text { type number }\end{array}$ & $\begin{array}{c}\text { Number of } \\
\text { tested } \\
\text { images }\end{array}$ & $\begin{array}{c}\text { Number of correctly } \\
\text { recognized maritime } \\
\text { objects }\end{array}$ & $\begin{array}{c}\text { Number of incorrectly } \\
\text { recognized maritime } \\
\text { objects }\end{array}$ & $\begin{array}{c}\text { Mean value of correctly } \\
\text { recognized maritime } \\
\text { objects }\end{array}$ \\
\hline \hline 1 & 14 & 14 & 0 & $100 \%$ \\
\hline 2 & 28 & 24 & 4 & $85,7 \%$ \\
\hline 3 & 16 & 16 & 0 & $100 \%$ \\
\hline 4 & 18 & 11 & 7 & $61,1 \%$ \\
\hline 5 & 10 & 10 & 0 & $100 \%$ \\
\hline 6 & 15 & 10 & 5 & $66,7 \%$ \\
\hline 7 & 9 & 5 & 4 & $55,6 \%$ \\
\hline Together & 110 & 90 & 20 & $81,8 \%$ \\
\hline \hline
\end{tabular}

\section{CONCLUSIONS}

The Table 4 compares the effectiveness of maritime object recognition based on FLIR images using the SVM classifier and the DTW classifier, and the Dempster's fusion of information classifiers. This comparison allows to positively assess the purposefulness of using the classifier fusion.

The classifier fusion increased the average effectiveness of the recognition of maritime objects from the level of $70,0 \%$ (SVM classifier) and 77,3\% (DTW classifier) to the level of $81,8 \%$. 
The results do not differ from the results presented in the studies using other segmentation methods and other classifiers $[13,15,16]$.

Table 4. Comparison of the effectiveness of recognizing images of maritime objects using SVM classifier, DTW classifier and the fusion of SVM and DTW classifiers by the Dempster's rule.

\begin{tabular}{|c||c|c|c|c||}
\hline $\begin{array}{c}\text { Maritime } \\
\text { object type } \\
\text { number }\end{array}$ & $\begin{array}{c}\text { Number of } \\
\text { tested images }\end{array}$ & $\begin{array}{c}\text { Effectiveness of the } \\
\text { SVM classifier }\end{array}$ & $\begin{array}{c}\text { Effectiveness of the } \\
\text { DTW classifier }\end{array}$ & $\begin{array}{c}\text { Effectiveness of Dempster's } \\
\text { fusion of SVM and DTW } \\
\text { classifiers }\end{array}$ \\
\hline \hline 1 & 14 & $50,0 \%$ & $100 \%$ & $100 \%$ \\
\hline 2 & 28 & $89,3 \%$ & $78,6 \%$ & $85,7 \%$ \\
\hline 3 & 16 & $56,3 \%$ & $100 \%$ & $100 \%$ \\
\hline 4 & 18 & $55,6 \%$ & $66,7 \%$ & $61,1 \%$ \\
\hline 5 & 10 & $60 \%$ & $100 \%$ & $100 \%$ \\
\hline 6 & 15 & $100 \%$ & $46,7 \%$ & $66,7 \%$ \\
\hline 7 & 9 & $55,6 \%$ & $44,4 \%$ & $55,6 \%$ \\
\hline \hline Together & 110 & $70,0 \%$ & $77,3 \%$ & $81,8 \%$ \\
\hline
\end{tabular}

Assessing the results of the SVM classifier, one should pay attention to the lower effectiveness of this classifier in the case of the first, third, fourth and seventh type of the recognized objects. The reason for such results is the inefficiency of this classifier to the changed aspect angles (different from the zero angle) under which the photos were taken. It is also necessary to analyze the reasons for the worse result of the DTW classifier in the case of the sixth and seventh types of objects.

Further directions of work in the field of recognition of maritime objects based on FLIR images may include the use of:

- better methods of image pre-processing, especially segmentation, e.g. using methods with multiple local thresholds or the unimodal-iterative method,

- other methods of decision making by SVM classifiers (SVM fusion) or nonlinear kernel functions in these classifiers,

- other methods of classifier fusion [22],

- deep neural networks.

Particularly the last point, and especially the convolutional neural networks, can improve the recognition of maritime objects based on FLIR images.

The results presented earlier allow to conclude that the work related to the recognition of maritime objects based on FLIR images should be continued and should lead to the development of an image recognition system cooperating with FLIR cameras in online mode.

\section{REFERENCES}

[1] Osowski, S, [Metody i narzędzia eksploracji danych], Wydawnictwo BTC, Legionowo (2013).

[2] Vapnik, V., [Statistical learning theory], Wiley, New York (1998).

[3] Vapnik, V., [The Nature of Statistical Learning Theory], 2 ed. Springer, New York (2000).

[4] Hsu, C.W., Chang, C.C., Lin, C.J., "A practical guide to support vector classification", 19 May 2016, $<$ https://www.csie.ntu.edu.tw/ cjlin/papers/guide/guide.pdf $>$ (10 December 2019).

[5] Hsu, C.W., Lin, C.J., "A comparison methods for multi class support vector machines", IEEE Trans. Neural Networks, vol. 13, 415-425 (2002).

[6] Burges, C.J.C., "A tutorial on support vector machines for pattern recognition, Data Mining and Knowledge Discovery”, June 1998, Volume 2, Issue 2, 121-167 (1998). 
[7] Gouaillieri, V., Gagnon, L., "Ship Silhouette Recognition Using Principal Components Analysis", SPIE Proc. \#3164, conference Applications of Digital Image Processing XX, San Diego, 59-69 (1997).

[8] Zhao, W., Chellappa, R., Phillips, P.J., Rosenfeld A., "Face Recognit,on: A Literature Survey", ACM Computing Surveys, Vol. 35, No. 4, 399-458 (2003).

[9] Matuszewski, J., "Identification of radar signals using discriminant vectors". XVII International Conference on Microwaves, Radar and Wireless Communications (MIKON), Wrocław, Poland, MAY 19-21, 2008, art. no. 4630190, 433-436 (2008).

[10] Gonzalez, R.C., Woods, R.E., [Digital Image Processing], Ed. 3, Pearson Prentice Hall (2008).

[11] Matuszewski, J., Zając, M., „Metody segmentacji obrazów w rozpoznawaniu cyfrowych zdjęć satelitarnych”, Przegląd Elektrotechniczny, vol. 95, nr 11, 2019, 113-116 (2019).

[12] Allen, S., "Signal Based Features with Application to Ship Recognition in FLIR Imagery", Proceedings of the 4th Annual Conference on Information Fusion, FUSION 2001, vol. 2, Montréal, Canada, August 7-10, 2001, FrC2-3 - FrC2-8 (2001).

[13] Jouan, A., Valin, P., Bossé, É., [Concepts of data/information fusion for naval C2 and airborne ISR platforms], Defence R\&D Canada - Valcartier, Technical Report DRDC Valcartier, TR 2004-284 (2006).

[14]Malina, W., Smiatacz, M., [Cyfrowe przetwarzanie obrazów], Akademicka Oficyna Wydawnicza EXIT, Warszawa (2008).

[15] Valin, P., "Methods for the Fusion of Multiple FLIR Classifiers", Proceedings of the Workshop on Signal Processing, Communication, Chaos and Systems: a Tribute to Rabinder N. Madan, June 20, 2002, Newport, RI, 117-122 (2002).

[16] Valin, P., Bossé É., Jouan A., [Airborne application of information fusion algorithms to classification], Defence R\&D Canada - Valcartier, Technical Report DRDC Valcartier, TR 2004-282 (2006).

[17] Müller, M., [Information Retrieval for Music and Motion], Springer-Verlag Berlin Heidelberg, 69-84 (2007).

[18] Teisseyre, P., „Porównanie szeregów czasowych z wykorzystaniem algorytmu DTW”, Instytut Podstaw Informatyki, Polska Akademia Nauk, 21 September 2010, <https://docplayer.pl/54095603-Porownanieszeregow-czasowych-z-wykorzystaniem-algorytmu-dtw.html> (10 December 2019).

[19]Fu, K.S., Mui, J.K., “A survey on image segmentation. Pattern Recognition”, 13, 3-16 (1981).

[20]Otsu, M., "A Threshold Selection Method from Gray-Level Histograms", IEEE Transactions SMC, No 1, $62-66$ (1979).

[21] Giorgino, T., "Computing and Visualizing Dynamic Time Warping Alignments in R: The DTW Package", Journal of Statistical Software, vol. 31, iss. 7 (2009).

[22] Smarandache, F., Dezert, J. (editors), [Advances and Applications of DSmT for Information Fusion (Collected works)], vol. 1, American Research Press, Rehoboth (2004).

[23]Phillips, P. J., "Support Vector Machines Applied to Face Recognition", Advances in Neural Information Processing Systems 11 (1998).

[24]Li, Y., Gong, S., Liddel, H., "Support Vector Regression and Classification Based Multi-view Face Detection and Recognition", Proceedings Fourth IEEE International Conference on Automatic Face and Gesture Recognition (Cat. No. PR00580), Grenoble, France, 300-305 (2000).

[25]Chapelle, O., Haffner, P., Vapnik, V., "Support Vector Machines for Histogram-Based Image Classification", IEEE Transactions on Neural Networks, vol. 10, no. 5, 1055-1064 (1999).

[26] Heisele, B., Ho, P., Poggio, T., "Face recognition with support vector machines: global versus component-based approach", Proceedings Eighth IEEE International Conference on Computer Vision. ICCV 2001, Vancouver, BC, Canada, 688-694 (2001).

[27] Matuszewski, J., "Radar signal identification using a neural network and pattern recognition methods", 14th International Conference on Modern Problems of Radio Engineering, Telecommunications and Computer Science, TCSET 2018 - Proceedings, 2018-April, Lviv-Slavske, Ukraine, 79-83 (2018).

[28] Chudy, M., [Wybrane metody optymalizacji], Bellona, Warszawa (2001).

[29] Pietkiewicz, T., "Application of fusion of two classifiers based on principal component analysis method and time series comparison to recognize maritime objects upon FLIR images", Proceedings of SPIE 11055, XII Conference on Reconnaissance and Electronic Warfare Systems, 110550Z (2019). 